\title{
CONHECIMENTO SOBRE MORTE ENCEFÁLICA E DOAÇÃO DE ÓRGÃO ENTRE ESTUDANTES DE MEDICINA DE BELO HORIZONTE
}

\author{
Knowledge on brain death and organ donation among medical students in Belo Horizonte
}

\author{
Ronaldo Eustáquio de Oliveira Júnior ${ }^{1}$, Bruno Oliveira Saldanha1, Ana Paula Pereira de Oliveira1, Eduardo Antunes dos Santos ${ }^{1}$, \\ Marcela Pereira Oliveira', Walter Antonio Pereira², Charles Simão Filho ${ }^{3}$
}

\begin{abstract}
RESUMO
Objetivo: Avaliar o conhecimento de estudantes de medicina dos $1^{\circ}, 6^{\circ}$ e $12^{\circ}$ períodos do curso sobre o protocolo de morte encefálica (ME) e doação de órgãos. Método: A partir de estudo descritivo transversal, um questionário com 25 questões foi aplicado a 248 estudantes de cinco faculdades de medicina de Minas Gerais no mês de junho de 2009 para aferir o conhecimento dos mesmos sobre os critérios de morte encefálica e doação de órgãos. Resultados: Dentre os entrevistados, 53,2\% afirmam não se lembrar de ter assistido à aula sobre ME durante o curso. Esse percentual é de apenas 25\%, se considerados apenas alunos do último ano. Encontrou-se prevalência de apenas 8,9\% de estudantes que afirmam conhecer muito bem sobre ME, sendo que a maioria (83,5\%) relata saber apenas um pouco sobre o tema. Mesmo entre os alunos do $12^{\circ}$ período, há desconhecimento a respeito dos reflexos pesquisados para protocolo de ME, assim como em relação aos exames complementares utilizados. No geral, trinta e dois por cento não sabem que um exame complementar é obrigatório e vinte por cento desconhecem a necessidade da participação de um neurologista. Apenas 70,6 \% de todos os pesquisados sabem que a notificação de ME é obrigatória. Em relação à doação de órgãos, 98,4\% de todos os estudantes afirmam ser favoráveis, 83,5\% desejam doar seus órgãos, enquanto 13,3\% afirmam nunca ter pensado no assunto. Entretanto, apenas $61,3 \%$ comunicaram à família a sua decisão sobre ser doador ou não. Conclusão: A atual pesquisa demonstra relativo desconhecimento dos estudantes em relação à ME. Há necessidade de valorizar mais esse tema durante a formação médica, pois esses estudantes conduzirão protocolos no futuro. Esse despreparo pode prejudicar a oferta de órgãos para a doação, aumentar o sofrimento de familiares, além de gerar gastos excessivos para hospitais.
\end{abstract}

Descritores: Morte Encefálica, Doação de Órgão, Transplante de Órgãos, Transplante de Tecidos, Estudantes de Medicina.

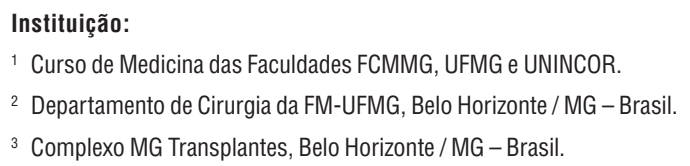

\section{INTRODUÇÃO}

A morte encefálica (ME) é definida como parada completa e irreversível das funções encefálicas, demonstrada pela presença de coma profundo, apnéia e nenhum reflexo supraespinhal. ${ }^{1} \mathrm{~A}$ ME deve ser consequência de processo irreversível e de causa conhecida em que se demonstre de forma inequívoca ausência de atividade cerebral. ${ }^{2}$

A não aceitação da morte por considerável parcela da população tem dificultado a definição dos limites terapêuticos nos cuidados intensivos. Também é comum a confusão entre os conceitos de $\mathrm{ME}$ e de paciente terminal ou em coma, sendo importante destacar que o primeiro está morto e, portanto, a suspensão das medidas de suporte de vida ou a doação de seus órgãos, nesse caso, podem ser realizadas sem prejuízo à autonomia da família ou do paciente. ${ }^{3}$ Os critérios para diagnóstico de ME estabelecidos a partir de 1959, envolvem parâmetros clínicos, e no Brasil, requerem a realização de exames complementares que demonstrem ausência de perfusão sanguínea cerebral ou da atividade elétrica ou da metabólica. Apesar de há bastante tempo este conceito já estar bem definido na comunidade científica mundial, ele ainda não é bem conhecido pela população geral e mesmo pela área médica., ${ }^{4,5}$ 
Há enorme discrepância entre o número de transplantes realizados e o de pacientes com doenças terminais à espera de um órgão. A demora na detecção de pacientes em ME pelos médicos pode levar à perda de um potencial doador pela piora dos parâmetros clínicos do paciente, que geralmente evolui com instabilidade cardiopulmonar e metabólica. ${ }^{6}$

Alguns estudos de diversos países já tentaram avaliar o conhecimento de estudantes de medicina sobre doação de órgãos e ME. ${ }^{7-13}$ Em estudo recente com acadêmicos de medicina de duas faculdades de Salvador/BA, observou-se que o conhecimento acerca de ME é limitado. A média de acertos para um questionário de 14 perguntas a respeitos dos critérios e abordagem de ME foi de 6,7 respostas corretas. Como o questionário foi aplicado em um evento científico extracurricular sobre medicina intensiva, com um viés de seleção, o resultado pode estar superestimando o que seria encontrado na população acadêmica em geral. ${ }^{3}$

Minas Gerais é um dos grandes centros transplantadores do Brasil e é de grande interesse avaliar qual o grau de conhecimento dos futuros médicos formados na região, já que nenhum estudo do tipo havia sido realizado no estado.

O objetivo do presente artigo é avaliar o conhecimento sobre ME entre estudantes de medicina da região metropolitana de Belo Horizonte durante o curso de graduação. Além disso, foi pesquisada a opinião sobre doação de órgão, assim como conhecimentos específicos a cerca do tema, como sua relação com o diagnóstico de morte encefálica.

\section{MÉTODOS}

Foi realizado estudo transversal dos estudantes de medicina das principais faculdades da região metropolitana de Belo Horizonte no início, meio e fim do curso. Após aprovação do Comitê de Ética em Pesquisa - CEP da Santa Casa de Misericórdia de Belo Horizonte foram avaliados estudantes do primeiro, sexto e décimo segundo períodos de cinco faculdades, sendo elas: Faculdade de Saúde e Ecologia Humana (FASEH), Faculdade de Ciências Médicas de Minas Gerais (FCMMG), Faculdade de Medicina da Universidade Federal de Minas Gerais (UFMG), Universidade do Vale do Rio Verde (UNINCOR) e Faculdade de Medicina do Vale do Aço.

Durante a primeira semana de junho de 2009 foi entregue um questionário autoaplicável contendo 25 perguntas que visavam avaliar o conhecimento e opinião sobre ME e doação de órgãos. Entre os assuntos pesquisados estavam: conceito e critérios de ME, exames complementares utilizados, intervalo entre exames clínicos, necessidade da participação de um neurologista e alguns detalhes e opiniões sobre a doação de órgãos. A confecção do questionário foi realizada após uma revisão da literatura científica, através de levantamento bibliográfico utilizando-se MEDLINE e LILACS como bancos de dados de consulta. A consulta nos referidos bancos de dados foi feita entre 1990 até 2009, utilizando-se os seguintes descritores: Morte Encefálica, Doação de Órgão, Transplante de Órgãos, Transplante de Tecidos, Estudantes de Medicina.

A distribuição dos questionários foi realizada nas salas em que os primeiro, sexto e décimo segundo períodos estivessem em aulas, sendo a participação na pesquisa voluntária e anônima. Nas instruções do questionário havia um termo de consentimento sobre a participação no estudo e publicação posterior dos resultados. Houve alguma dificuldade em encontrar estudantes do último ano do curso, uma vez que estes se encontravam principalmente realizando estágios práticos em diversos hospitais ao invés de reunidos em uma sala de aula. Após aplicação do questionário, foi distribuída cartilha informativa contendo cópia do termo de ME e as respostas para as perguntas teóricas realizadas.

A análise dos dados foi feita através do programa Epi Info na versão 6.04 e foi adotado o nível de significância estatística de 5\%. Na comparação de variáveis categóricas foi utilizado o qui-quadrado com correção de Yates.

\section{RESULTADOS}

Foram avaliados 248 estudantes de cinco faculdades de Medicina do estado de Minas Gerais que cursavam o primeiro, sexto e décimo segundo períodos, destes, 62,9\% eram do sexo feminino, sem muita diferença entre períodos. A maioria deles declarava-se da religião católica $(66,1 \%)$, seguido de $15,3 \%$ que não pertenciam a nenhuma religião (Tabela 1).

Tabela 1. Características Demográficas dos Estudantes Avaliados $(n=248)$

\begin{tabular}{lcccc}
\hline Variável & $\mathbf{1}^{\mathbf{0}}$ Período $\mathbf{n}(\%)$ & $\mathbf{6}^{\mathbf{0}}$ Período $\mathbf{n}(\%)$ & $\mathbf{1 2}^{\mathbf{0}}$ Período $\mathbf{n}(\%)$ & Total $\mathbf{n}(\%)$ \\
\hline Sexo & & & & \\
Masculino & $36(37,9)$ & $35(37,6)$ & $21(35,0)$ & $92(37,1)$ \\
$\quad$ Feminino & $59(62,1)$ & $38(62,4)$ & $39(65,0)$ & $156(62,9)$ \\
Faculdade & & & & \\
FASEH & $36(37,9)$ & $19(20,4)$ & $2(3,3)$ & $57(23,0)$ \\
FCMMG & $13(13,7)$ & $47(50,5)$ & $13(21,7)$ & $73(29,4)$ \\
FMUFMG & $25(26,3)$ & $24(25,8)$ & $29(48,3)$ & $78(31,5)$ \\
UNINCOR & $21(22,1)$ & $3(3,2)$ & $4(6,7)$ & $28(11,3)$ \\
IPATINGA & $0(0,0)$ & $0(0,0)$ & $12(20,0)$ & $12(4,8)$ \\
Religião & & & & \\
Católica & $55(57,9)$ & $65(69,9)$ & $44(73,3)$ & $164(66,1)$ \\
Evangélica & $9(9,5)$ & $8(8,6)$ & $2(3,3)$ & $19(7,7)$ \\
Espírita & $10(10,5)$ & $4(4,3)$ & $6(0,1)$ & $20(8,1)$ \\
Outra & $3(3,2)$ & $4(4,3)$ & $0(0,0)$ & $7(2,8)$ \\
Nenhuma & $18(18,9)$ & $12(12,9)$ & $8(13,3)$ & $38(15,3)$ \\
& & & & \\
\hline Total & 95 & 93 & 60 & 248 \\
\hline
\end{tabular}

FASEH = Faculdade de Saúde e Ecologia Humana: FCMMG = Faculdade de Ciências Médicas de Minas Gerais; FMUFMG = Faculdade de Medicina da Universidade Federal de Minas Gerais; UNINCOR = Universidade Vale do Rio Verde; IPATINGA = Faculdade de Medicina do Vale do Aço

Entre os entrevistados, 53,2\% não se lembraram de ter assistido a uma aula sobre morte encefálica, e esse percentual é de $25 \%$, se considerados somente alunos do ultimo ano. Apenas $8,9 \%$ dos estudantes afirmaram ter muito conhecimento sobre o tema, sendo que no $12^{\circ}$ período esse porcentual sobe apenas para $11,7 \%$. A pesquisa mostrou que $62,9 \%$ dos acadêmicos entrevistados consideram como critério para início da avaliação de ME a Escala de Coma de Glasgow $(E C G)=3$. Esse conhecimento evolui de 
forma surpreendente ao longo do curso, já que a porcentagem que respondeu corretamente a essa questão no $1^{\circ}, 6^{\circ}$ e $12^{\circ}$ períodos foi respectivamente de $37,9 \%, 71 \%$ e $90 \%$. Já em relação ao intervalo mínimo entre dois exames clínicos em pessoas acima de dois anos, a resposta que no geral foi a mais marcante foi a alternativa correta de seis horas $(34,7 \%)$. No entanto, vale ressaltar que a opção escolhida pela maioria dos alunos do último ano foi 12 horas com $45 \%$ contra $31,7 \%$ em relação à resposta certa. Sobre a participação de neurologista/neurocirurgião em pelo menos um exame clínico, $79,8 \%$ dos entrevistados sabia que esta era obrigatória. Chamamos a atenção para o fato que no último ano a resposta correta teve o menor percentual entre os períodos, com $66,7 \%$ contra $83,2 \%$ e $84,9 \%$ do $1^{\circ}$ e $6^{\circ}$ períodos respectivamente. Para $67,7 \%$ dos acadêmicos é obrigatória a realização de exames complementares, enquanto o restante ainda considera que é apenas um método auxiliar. Em relação à notificação de $\mathrm{ME}, 70,6 \%$ responderam que é obrigatória, $5,2 \%$ que não é e $24,2 \%$ afirmaram que não sabiam a resposta para a pergunta. A ME tem valor de morte clínica para $68,1 \%$ dos estudantes e percebe-se que esse foi um conhecimento adquirido ao longo do curso, uma vez que a resposta correta foi marcada por $52,6 \%$ do primeiro período, $75,3 \%$ do sexto e $81,7 \%$ do décimo segundo. Somam $81,5 \%$ os estudantes que confiam no diagnóstico de ME, sendo a desconfiança maior no início do curso $(12,6 \%)$ e mínima ao final $(3,3 \%)$. (Tabela 2$)$

Tabela 2. Avaliação do Conhecimento sobre Morte Encefálica $(n=248)$

\section{Variável}

$1^{0}$ Período n (\%) $6^{0}$ Período n (\%) $12^{\circ}$ Período n (\%) $\quad$ Total n (\%)

Já assistiu aula sobre ME? $(\mathrm{p}<0,0001)$

$\begin{array}{lcccc}\operatorname{Sim} & 35(36,8) & 36(38,7) & 45(75,0) & 116(46,8) \\ \text { Não } & 60(63,2) & 57(61,3) & 15(25,0) & 132(53,2) \\ \text { Conhece o conceito de ME? }(p=0,010) & & & \\ \text { Muito bem } & 3(3,2) & 12(12,9) & 7(11,7) & 22(8,9) \\ \text { Um pouco } & 79(83,2) & 77(82,8) & 51(85,0) & 207(83,5) \\ \text { Nada } & 13(13,7) & 4(4,3) & 2(3,3) & 19(7,7)\end{array}$

Qual paciente deve ser considerado para avaliação de ME? $(p<0,0001)$

$\begin{array}{lcccc}\text { Em coma } & 15(15,8) & 10(10,8) & 3(5,0) & 28(11,3) \\ \text { ECG }=3 & 36(37,9) & 66(71,0) & 54(90,0) & 156(62,9) \\ \text { ECG }<8 & 37(38,9) & 16(17,2) & 1(1,7) & 54(21,8) \\ \text { Coração parado } & 3(3,2) & 1(1,1) & 2(3,3) & 6(2,4) \\ \text { Em branco } & 4(4,2) & 0(0,0) & 0(0,0) & 4(1,6)\end{array}$

Qual deve ser 0 intervalo mínimo entre dois exames clínicos? $(p=0,019)$

$\begin{array}{lcccc}\text { Não há } & 21(22,1) & 7(7,5) & 4(6,7) & 32(12,9) \\ 3 \text { horas } & 13(13,8) & 13(14,0) & 3(5,0) & 29(11,7) \\ 6 \text { horas } & 28(29,5) & 39(41,9) & 19(31,7) & 86(34,7) \\ 12 \text { horas } & 25(26,4) & 23(24,7) & 27(45,0) & 75(30,2) \\ 24 \text { horas } & 8(8,4) & 11(11,8) & 7(11,7) & 26(10,5)\end{array}$

Participação de neurologista em um dos exames clínicos é obrigatória? ( $p=0,037)$

$\begin{array}{lllll}\text { Sim } & 79(83,2) & 79(84,9) & 40(66,7) & 198(79,8) \\ \text { Não } & 16(16,8) & 14(15,1) & 20(33,3) & 50(20,2)\end{array}$

Realização de exames complementares é obrigatória ou auxiliar? $(p=0,052)$

$\begin{array}{lllll}\text { Auxiliar } & 22(23,2) & 36(38,7) & 22(36,7) & 80(32,3) \\ \text { Obrigatória } & 73(76,8) & 57(61,3) & 38(63,3) & 168(67,7)\end{array}$

Notificação de ME é obrigatória? $(P=0,256)$

\begin{tabular}{|c|c|c|c|c|}
\hline Sim & $62(65,3)$ & $68(73,1)$ & $45(75,0)$ & $175(70,6)$ \\
\hline Não & $9(9,5)$ & $2(2,2)$ & $2(3,3)$ & $13(5,2)$ \\
\hline Não sei & $24(25,3)$ & $23(24,7)$ & $13(21,7)$ & $60(24,2)$ \\
\hline \multicolumn{5}{|c|}{ ME tem valor de morte clínica? $(p=0,0003)$} \\
\hline Sim & $50(52,6)$ & $70(75,3)$ & $49(81,7)$ & $169(68,1)$ \\
\hline Não & $45(47,4)$ & $23(24,7)$ & $11(18,3)$ & $79(31,9)$ \\
\hline \multicolumn{5}{|c|}{ Confia no diagnóstico de ME? $(p=0,118)$} \\
\hline $\operatorname{Sim}$ & $83(87,4)$ & $86(92,5)$ & $58(96,7)$ & $227(91,5)$ \\
\hline Não & $12(12,6)$ & $7(7,5)$ & $2(3,3)$ & $21(8,5)$ \\
\hline rotal & 95 & 93 & 60 & 248 \\
\hline
\end{tabular}

$M E=$ Morte Encefálica; $E C G=$ Escala de Coma de Glasgow

Dentre os critérios de avaliação clínica disponíveis no questionário que deveriam estar presentes em um paciente em ME, foram assinalados erroneamente por $18,1 \%$ dos alunos ausência de reflexo medular, por 10,1\% reflexo osteotendinoso ausente e por $12,9 \%$ o sinal de Babinski. A pupila paralítica foi o critério mais lembrado pelos estudantes, com $74,6 \%$. Interessante notar que respostas superiores a $50 \%$ se destacam somente em três itens: reflexo córneo-palpebral ausente, reflexo oculovestibular ausente e pupila paralítica. O período mais avançado obteve um maior índice de acerto em todos os critérios, com exceção de: arreatividade supra-espinhal e pupila paralítica. Frente aos exames que auxiliariam no diagnóstico de ME foram assinalados: EEG por 83,9\%, doppler transcraniano por $34,3 \%$, arteriografia $16,9 \%$, lembrando que no questionário era possível a marcação de mais de uma alternativa. A maioria dos estudantes conseguiu identificar pelo menos uma das condições que não devem estar presentes no exame de ME, sendo a sedação assinalada por $55,6 \%$ e a hipotermia por $39 \%$. (Tabela 3 )

Tabela 3. Avaliação do Conhecimento sobre os Critérios e Exames de ME

\begin{tabular}{cccccc}
\hline Variável & $1^{0}$ Período & $6^{0}$ Período & $12^{\circ}$ Período & Total & Valor $p$ \\
& $n(\%)$ & $n(\%)$ & $n(\%)$ & $n(\%) 10$ & \\
\hline
\end{tabular}

Qual(is) critérios deve(m) estar presente(s) na avaliação clínica de ME?

$\begin{array}{lccccc}\begin{array}{l}\text { - Apnéia* } \\ \text { - Arreatividade }\end{array} & 20(21,1) & 39(41,9) & 33(55,0) & 92(37,1) & <0,0001 \\ \begin{array}{l}\text { supra-espinhal* } \\ \text { - Reflexo corneopalpebral }\end{array} & 16(21,1) & 33(35,5) & 10(16,7) & 59(23,8) & 0,0036 \\ \text { ausente* } & 44(46,3) & 59(63,4) & 45(75,0) & 148(59,7) & 0,0012 \\ \text {-Reflexo de tosse ausente* }^{*} & 23(24,2) & 28(30,1) & 37(61,7) & 88(35,5) & <0,0001 \\ \text {-Reflexo medular ausente }^{*} & 21(22,1) & 16(17,2) & 8(13,3) & 45(18,1) & 0,369 \\ \text { - Reflexo oculovestibular } & & & & & \\ \text { ausente* } & 49(51,6) & 43(46,2) & 40(66,7) & 132(53,2) & 0,0432 \\ \text {-Reflexo oste0- } & & & & & \\ \text { tendinoso ausente } & 11(11,6) & 10(10,8) & 4(6,7) & 25(10,1) & 0,590 \\ \text {-Pupila paralítica* } & 60(63,2) & 78(83,9) & 47(78,3) & 185(74,6) & 0,003 \\ \text {-Sinal de Babinski } & 12(12,6) & 17(18,3) & 3(5,0) & 32(12,9) & 0,056\end{array}$


Qual(is) exame(s) ajudariam no diagnóstico de ME como exame complementar?

$\begin{array}{lccccc}\text {-Arteriografia* } & 13(13,7) & 18(19,4) & 11(18,3) & 42(16,9) & 0,553 \\ \text { - EEG* } & 71(74,7) & 84(90,3) & 53(88,3) & 208(83,9) & 0,008 \\ \text {-Exame Líquor } & 12(12,6) & 1(1,1) & 0(0,0) & 13(5,2) & 0,0002 \\ \text {-Doppler Transcraniano* } & 30(31,6) & 40(43,0) & 15(25,0) & 85(34,3) & 0,056 \\ \text {-TC } & 14(14,7) & 15(16,1) & 2(3,3) & 31(12,5) & 0,045 \\ \text {-ECG } & 9(9,5) & 3(3,2) & 2(3,3) & 14(5,6) & 0,120 \\ \text {-Nenhum } & 4(4,2) & 3(3,2) & 4(6,7) & 11(4,4) & 0,595\end{array}$

Qual(is) condição(ões) não deve(m) estar presente(s) durante exames de ME?

\begin{tabular}{lllllc}
-Hipernatremia* & $16(16,8)$ & $23(24,7)$ & $13(21,7)$ & $52(21,0)$ & 0,408 \\
-Hipotermia* & $30(31,6)$ & $44(47,3)$ & $23(38,3)$ & $97(39,0)$ & 0,086 \\
-Hipertermia & $27(28,4)$ & $23(24,7)$ & $13(21,7)$ & $63(25,4)$ & 0,631 \\
-Sedação* & $37(38,9)$ & $53(57,0)$ & $48(80,0)$ & $138(55,6)$ & $<0,0001$ \\
\hline
\end{tabular}

$M E=$ Morte Encefálica; * indica a resposta esperada

Em relação à doação de órgãos, 98,4\% dos entrevistados são a favor da doação, 83,5\% desejam doar seus órgãos, 89,5\% autorizariam a doação de órgãos de um parente e $61,3 \%$ já conversaram com a família sobre o desejo de ser ou não um doador. Vale ressaltar que $13,3 \%$ dos estudantes nunca pensaram sobre doar seus órgãos após a morte. (Tabela 4)

Tabela 4. Opinião dos Estudantes sobre a Doação de Órgãos

\begin{tabular}{|c|c|c|c|c|}
\hline $\begin{array}{l}\text { Variável } \\
\text { n(\%) }\end{array}$ & $\begin{array}{c}1^{0} \text { Período } \\
\text { n (\%) }\end{array}$ & $\begin{array}{c}6^{0} \text { Período } \\
\text { n (\%) }\end{array}$ & $\begin{array}{c}12^{\circ} \text { Período } \\
\text { n (\%) }\end{array}$ & Total \\
\hline \multicolumn{5}{|c|}{ Você é a favor da doação de órgãos? ( $p=0,161)$} \\
\hline Sim & $91(95,8)$ & $93(100,0)$ & $60(100,0)$ & $244(98,4)$ \\
\hline Não & $4(4,2)$ & $0(0,0)$ & $0(0,0)$ & $4(1,6)$ \\
\hline \multicolumn{5}{|c|}{ Você deseja doar seus órgãos após sua morte? $(p=0,115)$} \\
\hline $\operatorname{Sim}$ & $75(78,9)$ & $80(86,0)$ & $52(86,7)$ & $207(83,5)$ \\
\hline Não & $3(3,2)$ & $1(1,1)$ & $4(6,7)$ & $8(3,2)$ \\
\hline Nunca pensei & $17(17,9)$ & $12(12,9)$ & $4(6,7)$ & $33(13,3)$ \\
\hline \multicolumn{5}{|c|}{ Você autorizaria a doação de órgãos de um parente em ME? $(p=0,309)$} \\
\hline Sim & $81(85,3)$ & $84(90,3)$ & $57(95,0)$ & $222(89,5)$ \\
\hline Não & $14(13,7)$ & $9(9,7)$ & $3(5,0)$ & $26(10,5)$ \\
\hline \multicolumn{5}{|c|}{ Já comunicou sua família a sua decisão de ser ou não doador? $(P=0,043)$} \\
\hline Sim & $54(56,8)$ & $53(57,0)$ & $45(75,0)$ & $152(61,3)$ \\
\hline Não & $41(43,2)$ & $40(43,0)$ & $15(25,0)$ & $96(38,7)$ \\
\hline Total & 95 & 93 & 60 & 248 \\
\hline
\end{tabular}

$M E=$ Morte Encefálica

Foi questionado também como se dá a autorização da doação de órgãos e 50,8\% respondeu que seria necessário apenas que um parente assinasse a autorização, enquanto 9,7\% ainda pensa que é necessário ter por escrito que a pessoa é doadora de órgãos na carteira de identidade. O restante marcou que ambas as condições são necessárias $(p=0,056)$. Dentre os órgãos possíveis de serem doados por um paciente em ME, os que os estudantes mais se lembraram foi: coração $(94,4 \%)$, córnea $(92,3 \%)$, rim $(86,3 \%)$, fígado (80,6\%) e pulmão (79,4\%). Já o osso, intestino, pâncreas e pele foram marcados por menos da metade dos alunos, com 20,6\%, $29,8 \%, 43,1 \%$ e $45,2 \%$ respectivamente.

\section{DISCUSSÃO}

O presente estudo sugere que os estudantes do curso de Medicina majoritariamente $(98,4 \%)$ apóiam a doação de órgãos. Esse resultado é equiparável a outros estudos que demonstram que alunos daquele curso têm atitude a favor da doação de órgãos. ${ }^{8,9,14,15}$

Apesar disso, 13,3\% não desejam doar órgãos. A vontade de doar, assim como aqueles que autorizariam a doação de órgãos dos parentes, aumenta com o número de anos na faculdade. Isso pode sugerir que o conhecimento adquirido no curso pode converter a opinião de alguns alunos antes não-doadores, ou mesmo que o amadurecimento dessa idéia venha com a informação. Alguns estudos relacionam alto conhecimento sobre doação com aumento do interesse em doar. ${ }^{14,16,17}$ Esse resultado levanta hipótese de que talvez seja possível aumentar a doação de órgãos com campanhas educacionais sobre ME e doação de órgãos para a população. Se o conhecimento adquirido pelos estudantes aumenta sua vontade de doar órgãos, o mesmo pode ocorrer com todos os que estiverem mais inteirados do assunto.

Mesmo entre os alunos do $12^{\circ}$ período há desconhecimento a respeito de reflexos pesquisados para o protocolo de ME, assim como em relação aos exames complementares utilizados. No geral, apenas $67,7 \%$ afirmam ser obrigatória a realização de um exame complementar e $20 \%$ desconhecem a necessidade da participação de um neurologista. Apenas 70,6\% de todos os pesquisados sabem que a notificação de ME é obrigatória. Em estudo realizado na Bahia $^{3}$ em 2006 sobre o conhecimento entre estudantes médicos sobre transplantes e doação de órgãos, o achado foi de $80,7 \%$. Vale ressaltar ainda que os estudantes avaliados no estudo baiano compõem uma população selecionada, por estarem participando de um evento cientifico extracurricular sobre Medicina Intensiva, com possível viés de seleção.

Os educadores médicos deveriam incorporar temas de transplantes e doação de órgãos no atual currículo para formar profissionais da saúde capazes de reduzir a discrepância existente entre a oferta de órgãos e o número de pacientes à espera de um transplante.

Uma atitude favorável dos profissionais de saúde em relação à doação de órgãos pode influenciar positivamente a decisão de famílias de potenciais doadores. Através da melhoria do conhecimento desses profissionais sobre doação, transplantes e os qualificando para divulgar informações, a educação tem produzido uma resposta positiva para aumentar o número insuficiente de doadores. Educar os estudantes precocemente em suas carreiras pode se tornar crucial nessa conjuntura. ${ }^{18}$

Felizmente, o déficit no conhecimento dos intensivistas, em face do obrigatório protocolo que deve ser seguido não resulta no diagnóstico falso-positivo. Ou seja, não parece haver risco de algum paciente ter diagnóstico de morte encefálica sem de fato estar morto. Entretanto, há possibilidade de não se fazer o diagnóstico em pacientes que preenchem os critérios, o que causa além de outros danos ocupação desnecessária de um leito de UTI e indisponibilidade de captação de órgãos. 


\section{CONCLUSÃO}

Os dados deste trabalho confirmam os achados de estudos precedentes da falta de informação sobre ME entre estudantes de Medicina. Foi observado que durante o decorrer do curso, os alunos vão adquirindo conhecimentos sobre protocolo de $\mathrm{ME}$ e amadurecendo a idéia da doação de órgãos. No momento, este estudo comprova que são necessárias medidas que incluam em definitivo, no currículo das faculdades, uma matéria especifica de doação de órgãos e tecidos para transplantes, que possivelmente contribuirá para a formação de profissionais médicos aptos a conduzir o diagnóstico de morte encefálica.

\section{ABSTRACT}

Purpose: To assess the knowledge of medical students from the 1st, 6th and 12th semesters of the course on brain death and organ donation. Method: From a cross-sectional descriptive study, a questionnaire with 25 questions was applied to 248 students from 5 Medicine Schools in Minas Gerais along June 2009, to measure the knowledge of medical students on the brain death and organ donation criteria. Results: Among the respondents, 53.2\% affirm they don't remember attending a class on brain death along the course. Such percentage is only $25 \%$, when considering only students of the last year. It was found a prevalence of only $8.9 \%$ students who affirm to have a very good knowledge on brain death and the majority (83.5\%) reports to know just a little about the theme. Even among students from the 12 th semester, there is ignorance on the brain death criteria, as well as the supplementary exams. In general, thirty-two per cent do not know that a supplementary examination is mandatory, and twenty percent are unaware of the need for the participation of a neurologist. Only $70.6 \%$ of all respondents know that brain death notification is required. Related to the organ donation, 98.4 per cent of all students claim to be favorable, $83.5 \%$ want to donate their organs, while $13.3 \%$ say they have never thought about the subject. However, only $61.3 \%$ have reported to their family their decision as to be or not a donor. Conclusion: The current survey demonstrates a relative ignorance of students about brain death. There is need to enhance such theme along the medical training, since those students will lead these protocols in the future. This lack of knowledge may affect the organs supply for donation, thus increasing the suffering of relatives and causing excessive expenditure for hospitals.

Keywords: Brain Death; Organ Donation; Organ Transplantation; Tissue Transplantation; Medical, Students.

\section{REFERÊNCIAS}

1. Wijdicks EFM - The diagnosis of brain death. N Engl J Med. 2001;344:1215-21.

2. Parizi RR, Silma NM. Transplantes. In: Costa SIF, Garrafa V, Oselka G - Iniciacão Bioetica. Brasilia, Conselho Federal de Medicina. 1998;157-69.

3. Bitencourt AGV, Neves FBCS, Durães L, Nascimento DT, Neves NMBC, Torreão LA et al. Avaliação do Conhecimento de Estudantes de Medicina Sobre Morte Encefálica. RBTI. 2007;19:2:144-50.

4. Diagnosis of brain death: statement issued by the honorary secretary of the Conference of Medical Royal Colleges and their Faculties in the United Kingdom on 11 October 1976. Br Med J. 1976;2:1187-8.

5. Guidelines for the determination of death. Report of the medical consultants on the diagnosis of death to the President's Commission for the Study of Ethical Problems in Medicine and Biomedical and Behavioral Research. JAMA. 1981;246:2184-6.

6. Arbour R. Clinical Management of the Organ Donor. AACN Clinical Issues Advanced Practice in Acute \& Critical Care. 2005;16(4):551-580.

7. Bagheri A, Tanaka T, Takahashi H, Shoji S. Brain death and organ transplantation knowledge, attitudes, and practice among Japanese students. Eubios J Asian Int Bioeth. 2003;13:3-5

8. Bardell T, Hunter DJ, Kent WD, Jain MK. Do medical students have the knowledge needed to maximize organ donation rates? Can J Surg. 2003;46:453-7.

9. Dutra MM, Bonfim TA, Pereira IS, Figueiredo IC, Dutra AMD, Lopes AA1. Knowledge about transplantation and attitudes toward organ donation: a survey among medical students in northeast Brazil. Transplant Proc. 2004;36:818-20.
10. Essman C, Thornton J. Assessing medical student knowledge, attitudes, and behaviors regarding organ donation. Transplant Proc. 2006;38:2745-50

11. Ohwaki K, Yano E, Shirouzu M, Kobayashi A, Nakagomi T, Tamura A. Factors associated with attitude and hypothetical behaviour regarding brain death and organ transplantation: comparison between medical and other university students. Clin Transplant. 2006;20:416-22.

12. Laederach-Hofmann K, Gerster BI. Knowledge, attitude and reservations of medical students about organ transplantation: results of a survey during the first year of study. Schweiz Med Wochenschr. 1998;128:1840-9.

13. Afonso RC, Buttros DA, Sakabe D, Paranhos GC, Garcia LMC, Resende MB. Future doctors and brain death: what is the prognosis? Transplant Proc. 2004;36:816-7.

14. Schaeffner ES, Windisch W, Freidel K, Breitenfeldt K, Winkelmayer WC. Knowledge and attitude regarding organ donation among medical students and physicians. Transplantation. 2004; 77:1714.

15. Cantwell M, Clifford C. English nursing and medical students' attitudes towards organ donation. J Adv Nurs. 2000; 32:961.

16. Thornton JD, Wong KA, Cardenas V, Curtis JR, Spigner C, Allen MD. Ethnic and gender differences in willingness among high school students to donate organs. J Adolesc Health. 2006; 39:266.

17. Siminoff LA, Burant C, Youngner SJ. Death and organ procurement: public beliefs and attitudes. Soc Sci Med. 2004; 59:2325.

18. Garcia CD, Barboza AP, Goldani JC, Neumann J, Chem R, Camargo J et al. Educational Program of Organ Donation and Transplantation at Medical School. Transplant Proc. 2008; 40:1068-9. 